\title{
Enantioselective Determination of Synephrine in Health Food Products by Liquid Chromatography/Time-of-Flight Mass Spectrometry
}

\author{
Yoshiyuki KOBAYASHI, Masakadzu YATO, Rie ITO, Koichi SAITO* \\ Department of Analytical Chemistry, Faculty of Pharmaceutical Sciences, Hoshi University, 2-4-41, Ebara, Shinagawa-ku,
}

Tokyo 142-8501, Japan

\begin{abstract}
An enantioselective analytical method for the determination of synephrine, an ingredient in health food, by liquid chromatography/time-of-flight mass spectrometry (LC/TOF-MS) was developed. By derivatizing synephrine with FMOC and using TCI Chiral MB-S as the chiral column, FMOC-synephrine enantiomers were well separated. The electrospray ionization (ESI) negative mode was adopted for TOF-MS measurement. Good linearity $(r>0.999)$ was obtained in the concentration range of 0.5 to $50 \mu \mathrm{g} / \mathrm{mL}$. The limit of detection (LOD, $S / N=3$ ) and the limit of quantification (LOQ, $S / N>$ 10) were $0.25 \mu \mathrm{g} / \mathrm{mL}$ and $0.5 \mu \mathrm{g} / \mathrm{mL}$, respectively. The intra-day and inter-day accuracy of synephrine enantiomers at the LOQ level $(0.5 \mu \mathrm{g} / \mathrm{mL})$, the intermediate concentration level $(10 \mu \mathrm{g} / \mathrm{mL})$, and the high concentration level $(50 \mu \mathrm{g} / \mathrm{mL})$ ranged from 90.0 to $107.4 \%$. The intra-day and inter-day precisions were $\leq 9.24 \%$ and $\leq 10.63 \%$, respectively. As a result of analyzing synephrine-containing health foods using this method, approximately half of the products showed high optical purity with $80-100 \%$ enantiomeric excess of the $l$-isomer. In contrast, nearly $20 \%$ of the products contained racemate with $0-50 \%$ enantiomeric excess, and variations in optical purity were observed for the products. It was speculated that the $l$-isomer could be converted into the $d$-isomer during the manufacturing process.
\end{abstract}

Keywords: Synephrine; LC/TOF-MS; Enantioselective determination; Enantiomer; Health food; FMOC-Cl

\section{Introduction}

Synephrine (Fig. 1) is an alkaloid contained in bitter orange (Citrus aurantium) [1] and is said to have fat-burning effects [2]. In recent years, C. aurantium extract has been used in slimming health foods as an alternative to ephedrine-containing supplements that were banned by the US FDA in 2004 [3]. Synephrine has been reported to have thermogenic and lipolytic effects by acting non-selectively on adrenergic receptors, but it has also been reported to affect the cardiovascular system. There are concerns about its side effects, such as high blood pressure and arrhythmia $[4,5]$.

Synephrine has an asymmetric center, and it is known that the $l$-isomer is predominantly present in natural products [6,7]. Because the $l$-isomer acts on adrenergic receptors 10 to 100 times more strongly than the $d$-isomer $[8,9]$, the optical purity may affect such side effects as high

*Corresponding author: Koichi SAITO

Tel: +81-3-5498-5764; Fax: +81-3-5498-5764

E-mail:k-saito@hoshi.ac.jp blood pressure and arrhythmia. Therefore, it is necessary to measure the optical purity as well as the synephrine content in food supplements from the perspective of food hygiene and safety.

Previously, the enantioselective separation of synephrine was performed by chiral ligand exchange chromatography [10-12]. Because this method requires the addition of copper sulfate or EDTA to the mobile phase, it is difficult to use a mass spectrometer (MS) as the detector. Hence, a UV detector has been mainly used [13-18]. However, as the absorption maximum of synephrine appears in the low wavelength region of 210 to $225 \mathrm{~nm}$ and not the high wavelength region, it is considered that selectivity is low and influenced by impurities in food. Furthermore, as synephrine is a highly polar substance, it is weakly retained on a reversed-phase column, and interaction with the chiral stationary phase is considered to be weak. For these reasons,

Received: 2 October 2019

Accepted: 14 November 2019

J-STAGE Advance Published: 24 November 2019

DOI: $10.15583 /$ jpchrom.2019.024 
enantioselective separation is considered difficult.

In this study, we adopted 9-fluorenylmethyl chloroformate (FMOC-Cl) (Fig. 1) as the derivatization reagent to derivatize synephrine. By derivatizing synephrine, we could improve column retention and enantioselective separation by a chiral column. In addition, considering the effects of impurities in the food matrix, we developed an enantioselective analytical method for synephrine that uses a liquid chromatograph/time-of-flight mass spectrometer (LC/TOF-MS) with high selectivity.

The quality of commercial synephrine-containing health food products was evaluated by measuring the optical purity of synephrine using the developed analytical method.<smiles>CNC[C@H](O)c1ccc(O)cc1</smiles>

l-Synephrine<smiles>CNC[C@@H](O)c1ccc(O)cc1</smiles><smiles>O=C(Cl)OCC1c2ccccc2-c2ccccc21</smiles>

FMOC-Cl<smiles>CCCCCCCCCCCCCCCOC(=O)N(C)CC(O)c1ccc(O)cc1</smiles>

Fig. 1. Chemical structures of synephrine, FMOC-Cl and FMOC-synephrine.

\section{Experimental}

\subsection{Materials and reagents}

A total of 28 health food products (capsules and tablets ranged from $0.20 \mathrm{~g}$ to $1.59 \mathrm{~g}$ ) claiming to contain $C$. aurantium or synephrine were selected as measurement samples. An extract of commercially available Satsuma mandarin (Citrus unshiu Marc.) was used for the identification of the $l$-isomer.

Synephrine analytical standard (purity $>98 \%$, racemate) was purchased from FUJIFILM Wako Pure Chemical Corporation. As the derivatization reagent, 9-fluorenylmethyl chloroformate (FMOC-Cl) (purity > 98\%) was purchased from Tokyo Chemical Industry Co., Ltd. HPLC-grade methanol and acetonitrile, Wako super special-grade acetic acid and formic acid, special-grade ammonium acetate, ammonium formate, ammonium carbonate, sodium hydroxide, ammonia water, triethylamine, and biochemical-grade leucine-enkephalin were purchased from FUJIFILM Wako Pure Chemical Corporation. Ultrapure water was obtained with a Milli-Q Gradient A10 system equipped with an $\operatorname{EDS}-\mathrm{PAK}(\mathrm{R})$ polisher (Merck Millipore Ltd., Bedford, MA, USA).

\subsection{Preparation of calibration solution}

A $0.25 \mu \mathrm{g} / \mathrm{mL}$ leucine-enkephalin solution was prepared in $50 \%$ aqueous acetonitrile solution containing $0.1 \%$ formic acid, and stored in a refrigerator.

\subsection{Preparation of standard solutions}

To prepare a standard stock solution $(1.0 \mathrm{mg} / \mathrm{mL})$, we weighed precisely $10 \mathrm{mg}$ of synephrine analytical standard and dissolved it in $10 \mathrm{~mL}$ of purified water. We diluted this solution with an appropriate volume of purified water for use as the standard solution. The concentration of FMOC-Cl was adjusted to $15 \mathrm{mM}$ with acetonitrile and used as the derivatization reagent. The standard stock solution and the standard solution were stored at $4^{\circ} \mathrm{C}$ or lower until use.

\subsection{FMOC derivatization reaction}

To $250 \mu \mathrm{L}$ of synephrine standard solution, $250 \mu \mathrm{L}$ of the $15 \mathrm{mM}$ FMOC-Cl acetonitrile solution as the derivatization reagent and $50 \mu \mathrm{L}$ of $1 \mathrm{M}$ ammonia solution were added as the reaction solvent. The mixed solution was stirred for 40 seconds and the volume was adjusted to $10 \mathrm{~mL}$ with $50 \%$ aqueous acetonitrile solution to obtain the measurement solution.

\subsection{Preparation of sample solution}

For tablets and capsules, we measured the weight of one tablet or capsule taken out from the bottle. In the case of a tablet, the tablet was crushed in a mortar and pestle in advance. In the case of a hard capsule, the capsule shell was separated and the contents were taken out. In the case of a soft capsule, a cut of approximately several mm was made at the tip with a knife, and the contents were taken out. Then, purified water was added to each of them to make 50 $\mathrm{mL}$ and ultrasonic extraction was performed for 20 minutes. The solution was filtered through $0.2 \mu \mathrm{m}$ membrane filter to obtain the sample solution.

For Satsuma mandarin, after removing the peel from whole fruit, the fruit was squeezed and approximately $5 \mathrm{~mL}$ of juice was collected. The juice was filtered through 0.2 $\mu \mathrm{m}$ membrane filter to obtain the sample solution for the identification of enantiomers. FMOC derivatization was performed according to the procedure in "2.4. FMOC derivatization reaction" using $250 \mu \mathrm{L}$ of the sample 
solution.

\subsection{Apparatus and operating conditions}

An Alliance HT 2795 equipped with LCT Premier XE (Waters Corporation) was used for LC/TOF-MS. TCI Chiral MB-S (250 mm $\times 4.6 \mathrm{~mm}$ i.d., $5 \mu \mathrm{m}$, Tokyo Chemical Industry Co., Ltd.) with optically active maleimide polymer as the stationary phase was used at $30^{\circ} \mathrm{C}$. Acetonitrile- 10 $\mathrm{mM}$ acetic acid aqueous solution $(30: 70, \mathrm{v} / \mathrm{v})$ as the mobile phase was used at the flow rate of $0.5 \mathrm{~mL} / \mathrm{min}$. The negative ion mode of the electrospray ionization (ESI) method was adopted for ionization in TOF-MS. The lock mass method was used for the correction of accurate mass, and $50 \%$ aqueous acetonitrile solution containing $0.1 \%$ formic acid was used as the standard substance for mass calibration at $0.25 \mu \mathrm{g} / \mathrm{mL}$ leucine-enkephalin $\left([\mathrm{M}+\mathrm{H}]^{+}\right.$: 556.2771). Details of other measurement conditions appear in Table 1. AP-1020 polarimeter (JASCO Corporation) was used to measure the optical rotation of FMOC-synephrine.

Table 1. Operating conditions of LC/TOF-MS for the determination of FMOC-synephrine.

\begin{tabular}{ll}
\hline LC conditions & Alliance HT 2795 \\
Instrument & TCI Chiral MB-S \\
Column & $(250 \mathrm{~mm} \times 4.6 \mathrm{~mm}$ I.D., $5 \mu \mathrm{m})$ \\
Mobile phase A & Acetonitrile \\
Mobile phase B & $10 \mathrm{mM}$ Acetic acid \\
Flow rate & $0.5 \mathrm{~mL} / \mathrm{min}(\mathrm{A}: \mathrm{B}=30: 70)$ \\
Column temperature & $30^{\circ} \mathrm{C}$ \\
Injection volume & $20 \mu \mathrm{L}$ \\
& \\
MS conditions & \\
Instrument & $\mathrm{LCT}$ Premier XE \\
Ionization mode & $\mathrm{ESI} \mathrm{negative}$ \\
Capillary voltage & $2000 \mathrm{~V}$ \\
Aperture \# 1 voltage & $15 \mathrm{~V}$ \\
Cone voltage & $50 \mathrm{~V}$ \\
Desolvation temperature & $350^{\circ} \mathrm{C}$ \\
Source temperature & $120^{\circ} \mathrm{C}$ \\
Desolvation gas flow $\left(\mathrm{N}_{2}\right)$ & $750 \mathrm{~L} / \mathrm{h}$ \\
Cone gas flow $\left(\mathrm{N}_{2}\right)$ & $50 \mathrm{~L} / \mathrm{h}$ \\
\hline
\end{tabular}

\subsection{Method validation for LC/TOF-MS}

To confirm the validity of the developed analytical method, the linearity, the limit of detection (LOD), and the limit of quantification (LOQ) were calculated using the standard solution. The linearity of the detector response was demonstrated by the determination of calibration standards using an absolute calibration curve method at a minimum of six concentration levels ranging from $0.5 \mu \mathrm{g} / \mathrm{mL}$ to 50 $\mu \mathrm{g} / \mathrm{mL}(0.5,1,5,10,25,50 \mu \mathrm{g} / \mathrm{mL})$. A portion of the health food products was used as the sample in a recovery test. Measurement was repeated five times per day and repeated for three days. The obtained data were statistically analyzed by one-way analysis of variance, and the intra-day and inter-day accuracy and precision were calculated.

\section{Results and discussion}

\subsection{Investigation of FMOC derivatization of synephrine}

The separation of enantiomers is generally said to require three or more simultaneous reversible interactions between the enantiomers and the chiral stationary phase [19]. As synephrine is a highly polar substance, it is weakly retained on a reversed-phase column, its interaction with a chiral stationary phase is considered to be weak, and optical separation under reversed-phase conditions is difficult. Thus, we attempted to perform an indirect enantiomeric separation by derivatizing the target substance and changing its physical properties. In conventional indirect separation methods, a diastereomer is formed by using an optically active derivatization reagent, and separation is performed with a non-chiral column, such as ODS [10-12].

However, in those methods, the optical purity of the derivatization reagent is often unknown and it is necessary to calculate the optical purity separately. Thus, we adopted an enantioselective analytical method in which the target substance is derivatized with a reagent that does not have optical activity. We attempted to change the physical properties of the target substance and use a chiral column [20-22]. FMOC-Cl was assessed as the derivatization reagent. FMOC-Cl selectively reacts with primary amines and secondary amines, and the reaction proceeds easily by stirring for several tens of seconds at room temperature. Because synephrine isomerizes when heated for a long time [23], we adopted FMOC-Cl, which is reactive under mild conditions. The FMOC derivatization reaction is a kind of Schotten-Baumann reaction, and hydrogen chloride is generated during the reaction process [24]. Basic substances are used to neutralize hydrogen chloride. The reaction rate was confirmed by changing the concentration of each of the four basic solvents: ammonia aqueous solution, triethylamine aqueous solution, ammonium carbonate aqueous solution, and sodium hydroxide aqueous solution, as reaction solvents. As a result, the highest reaction rate was obtained in $1 \mathrm{M}$ ammonia aqueous solution. When the reaction rate in $1 \mathrm{M}$ ammonia aqueous solution was $100 \%$, the reaction rate in ammonium carbonate aqueous solution was approximately $65 \%$ and that in triethylamine aqueous solution was approximately $50 \%$. No reaction was observed in sodium hydroxide aqueous solution. A calibration curve was prepared using $1 \mathrm{M}$ ammonia aqueous solution, and good linearity was obtained in the range of 0.5 to $50 \mu \mathrm{g} / \mathrm{mL}$ $(r=0.999)$. Therefore, $1 \mathrm{M}$ ammonia aqueous solution was used as the reaction solvent. 


\subsection{Optimization of LC/TOF-MS operating conditions}

We examined four stationary phases of CHIRALPAK AD-R, CHIROBIOTIC T, CHIRALCEL OA, and TCI Chiral MB-S for LC separation on a chiral column. When TCI Chiral MB-S with optically active maleimide polymer was used as the stationary phase, good enantioselective separation of FMOC-synephrine was achieved $(R s=3.1)$. In contrast, synephrine enantiomers were not separated in the other three columns.

As regards the mobile phase, retention times and peak areas were compared among formic acid, ammonium formate, acetic acid, and ammonium acetate at $10 \mathrm{mM}$. The best peak area was obtained in both ESI positive and negative ion modes by using acetic acid. In the positive ion mode, when the peak area when acetic acid was used was $100 \%$, peak areas of approximately $60 \%$ for formic acid and ammonium formate and approximately $35 \%$ for ammonium acetate were obtained. In the negative ion mode, formic acid and ammonium formate had peak areas of approximately $5 \%$ relative to acetic acid. Almost no ionization was observed with ammonium acetate. There was no significant difference in the retention time among any buffer solutions and acids.

The ionization mode for TOF-MS measurement of FMOC-synephrine was examined, and good response was obtained when acetic acid was used as the mobile phase in both ESI positive and negative ion modes. The difference in the relative ratios of peak areas in each ion mode was within 5\%. However, when background noise was compared, in the positive ion mode, many subtle noise peaks with relative ratios of approximately 10 to $40 \%$ were detected in the range of $\mathrm{m} / \mathrm{z} 100$ to 300 and $\mathrm{m} / \mathrm{z} 300$ to 500 . In contrast, in the negative ion mode, background noise detected in the positive ion mode was not detected. Thus, we adopted the negative ion mode with low background noise. Table 1 shows the other MS conditions.

The mass spectrum of synephrine standard solution (10 $\mu \mathrm{g} / \mathrm{mL}$ ) measured by this method is shown in Fig. 2 .

\subsection{Identification of synephrine enantiomers}

Synephrine analytical standard that can be purchased from reagent manufacturers was only the racemate, and no analytical standard of enantiomer is available. Thus, in order to identify the enantiomers in the two peaks detected as FMOC-synephrine, we used Satsuma mandarin, which has a significantly high content of the $l$-isomer.

The juice of Satsuma mandarin was derivatized by FMOC and used in LC/TOF-MS measurement. A peak with a long retention time (19.20 minutes) was visible in the mass chromatogram (Fig. 3). Fractions corresponding to this peak were collected several times and optical rotation was measured with a polarimeter using $5 \mathrm{mg}$ equivalent as synephrine. As negative optical rotation $([\alpha]:-85.40 \mathrm{deg})$ was obtained, the peak with a long retention time (19.20 minutes) was identified as $l$-synephrine, and that with a shorter retention time (17.59 minutes) was identified as $d$-synephrine.

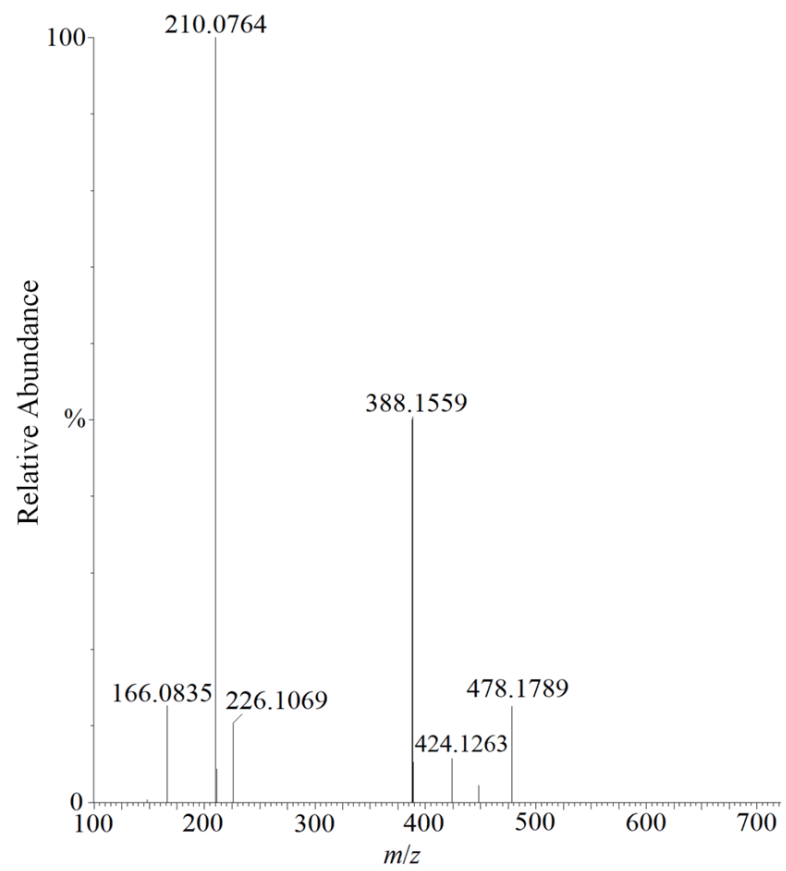

Fig. 2. Mass spectrum of FMOC-synephrine.

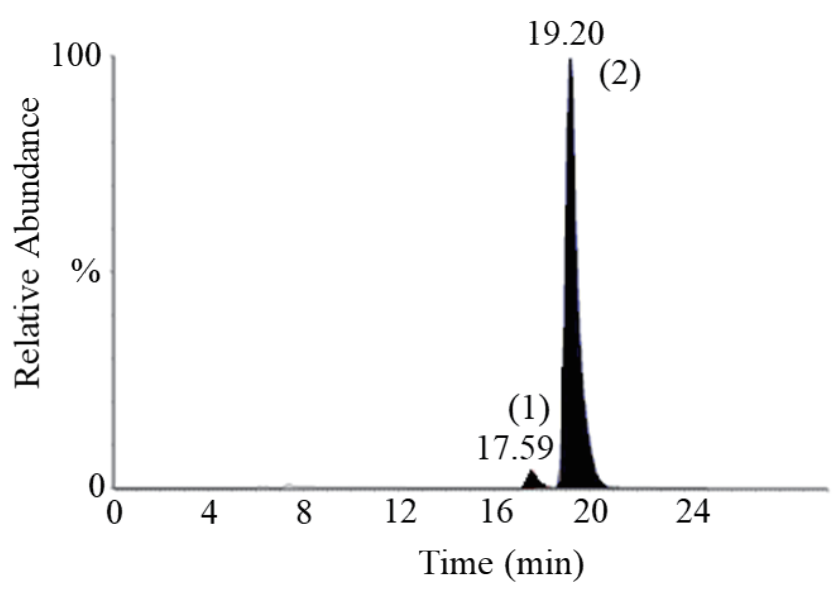

Fig. 3. Representative mass chromatogram of FMOC-synephrine extracted from Satsuma mandarin. Peak (1): FMOC- $d$-synephrine, Peak (2): FMOC-l-synephrine.

\subsection{Method validation}

LOD and LOQ were determined. Using working solutions with different concentrations, a calibration curve was prepared. The calibration curve of FMOC-synephrine at least six concentration levels showed good linearity $(r>$ $0.999)$ in the range of 0.5 to $50 \mu \mathrm{g} / \mathrm{mL}(0.5,1,5,10,25,50$ $\mu \mathrm{g} / \mathrm{mL})$. LOD $(S / N=3)$ and LOQ $(S / N>10)$ were 0.25 $\mu \mathrm{g} / \mathrm{mL}$ and $0.5 \mu \mathrm{g} / \mathrm{mL}$, respectively (Table 2 ). 
Table 2. Method validation of the proposed LC/TOF-MS analysis.

\begin{tabular}{ccccc}
\hline & $\begin{array}{c}\text { LOD } \\
(\mu \mathrm{g} / \mathrm{mL})\end{array}$ & $\begin{array}{c}\text { LOQ } \\
(\mu \mathrm{g} / \mathrm{mL})\end{array}$ & $\begin{array}{c}\text { Range } \\
(\mu \mathrm{g} / \mathrm{mL})\end{array}$ & $\begin{array}{c}\text { Correlation } \\
\text { coefficient }(r)\end{array}$ \\
\hline$d$-form & 0.25 & 0.5 & $0.5 \sim 50$ & 0.999 \\
$l$-form & 0.25 & 0.5 & $0.5 \sim 50$ & 0.999 \\
\hline
\end{tabular}

\subsection{Recovery test}

Recovery tests were performed using synephrine-free health food as sample. Synephrine standard solution was added to the health food at low concentration level $(0.5$ $\mu \mathrm{g} / \mathrm{mL})$, intermediate concentration level $(10 \mu \mathrm{g} / \mathrm{mL})$, and high concentration level $(50 \mu \mathrm{g} / \mathrm{mL})$. Five replicate determinations for each concentration were carried out for three days. The intra-day and inter-day accuracy at the low concentration level ranged from 92.5 to $106.5 \%$, that at the intermediate concentration level ranged from 90.0 to $96.4 \%$, and that at the high concentration level ranged from 99.5 to $107.4 \%$. The intra-day and inter-day precisions were within $9.24 \%$ and $9.46 \%$ at the low concentration level, within $8.27 \%$ and $10.63 \%$ at the intermediate concentration level, and within $4.77 \%$ and $9.63 \%$ at the high concentration level (Table 3).

Table 3. Intra-day and inter-day accuracy and precision of FMOC-synephrine in health foods.

\begin{tabular}{cccccc}
\hline & $\begin{array}{c}\text { Spiked } \\
\text { amount } \\
(\mu \mathrm{g} / \mathrm{mL})\end{array}$ & $\begin{array}{c}\text { Accuracy } \\
(\%)\end{array}$ & $\begin{array}{c}\text { Precision } \\
(\%)\end{array}$ & $\begin{array}{c}\text { Accuracy } \\
(\%)\end{array}$ & $\begin{array}{c}\text { Precision } \\
(\%)\end{array}$ \\
\cline { 3 - 6 } & 0.5 & 106.57 & 9.24 & 101.97 & 9.46 \\
$\begin{array}{c}d- \\
\text { form }\end{array}$ & 10 & 94.56 & 8.27 & 90.00 & 9.31 \\
& 50 & 100.50 & 4.71 & 107.43 & 9.01 \\
& & & & & \\
$l-$ & 10 & 96.5 & 2.99 & 92.59 & 3.17 \\
form & 50 & 99.57 & 4.77 & 107.31 & 9.63 \\
\hline
\end{tabular}

$(n=5,3$ days $)$

\subsection{Application to commercial health foods}

This method was applied to the analysis of imported and local health food products (28 in total) that claim to contain synephrine, and optical purity was measured respectively. The representative chromatogram is shown in Fig. 4, and the measurement results are shown in Table 4.

Among the 28 health food products, 12 (approximately $40 \%$ ) showed high optical purity with 80 to $100 \%$ enantiomeric excess of the $l$-isomer. These products indeed contained naturally occurring synephrine as indicated on the product labels.

In contrast, seven health food products (approximately
$20 \%$ ) showed an enantiomeric excess of approximately 0 to $50 \%$, indicating a racemate. Variations in optical purity were also confirmed for these products. Although there were some health food products that claim to contain $l$-synephrine derived from $C$. aurantium extract, a part of those products was revealed to contain racemates. That is, the presence of racemates indicates that synthetic synephrine compound was used in the health food products instead of $C$. aurantium extract. Furthermore, it has been reported that synephrine is isomerized when heated for a long time [24]. Therefore, it is speculated that the $l$-isomer was converted into the $d$-isomer during the manufacturing process of health foods.

Table 4. Optical purity of synephrine in health foods.

\begin{tabular}{|c|c|c|c|c|}
\hline \multirow[t]{2}{*}{ No. } & \multirow{2}{*}{$\begin{array}{l}\text { Content } \\
(\mathrm{mg} / \mathrm{g})\end{array}$} & \multicolumn{2}{|c|}{$\begin{array}{l}\text { Optical purity } \\
\text { measured, \% }\end{array}$} & \multirow[t]{2}{*}{$e e, \%$} \\
\hline & & $l$ & $d$ & \\
\hline 1 & 90.4 & 67.4 & 32.6 & 34.8 \\
\hline 2 & 93.0 & 66.2 & 33.8 & 32.4 \\
\hline 3 & 47.1 & 96.4 & 3.6 & 92.8 \\
\hline 4 & 42.1 & 82.1 & 17.9 & 64.2 \\
\hline 5 & 5.0 & 95.1 & 4.9 & 90.2 \\
\hline 6 & 25.0 & 50.1 & 49.9 & 0.2 \\
\hline 7 & 18.9 & 93.6 & 6.4 & 87.2 \\
\hline 8 & 14.8 & 92.6 & 7.4 & 85.2 \\
\hline 9 & 11.2 & 89.2 & 10.8 & 78.4 \\
\hline 10 & 16.0 & 50.1 & 49.9 & 0.2 \\
\hline 11 & 87.9 & 50.0 & 50.0 & 0 \\
\hline 12 & 14.3 & 89.1 & 10.9 & 78.2 \\
\hline 13 & 6.0 & 95.1 & 4.9 & 90.2 \\
\hline 14 & 13.8 & 94.5 & 5.5 & 89.0 \\
\hline 15 & 14.2 & 92.8 & 7.2 & 85.6 \\
\hline 16 & 5.0 & 89.5 & 10.5 & 79.0 \\
\hline 17 & 6.1 & 94.3 & 5.7 & 88.6 \\
\hline 18 & 3.8 & 82.0 & 18 & 64.0 \\
\hline 19 & 14.4 & 99.8 & 0.2 & 99.6 \\
\hline 20 & 14.0 & 92.3 & 7.7 & 84.6 \\
\hline 21 & 7.2 & 92.6 & 7.4 & 85.2 \\
\hline 22 & 34.5 & 50.0 & 50.0 & 0 \\
\hline 23 & 8.0 & 89.3 & 10.7 & 78.6 \\
\hline 24 & 1.0 & 95.1 & 4.9 & 90.2 \\
\hline 25 & 33.5 & 54.3 & 45.7 & 8.6 \\
\hline 26 & 43.1 & 86.1 & 13.9 & 72.2 \\
\hline 27 & 61.5 & 88.9 & 11.1 & 77.8 \\
\hline 28 & 0.8 & 86.4 & 13.6 & 72.8 \\
\hline
\end{tabular}




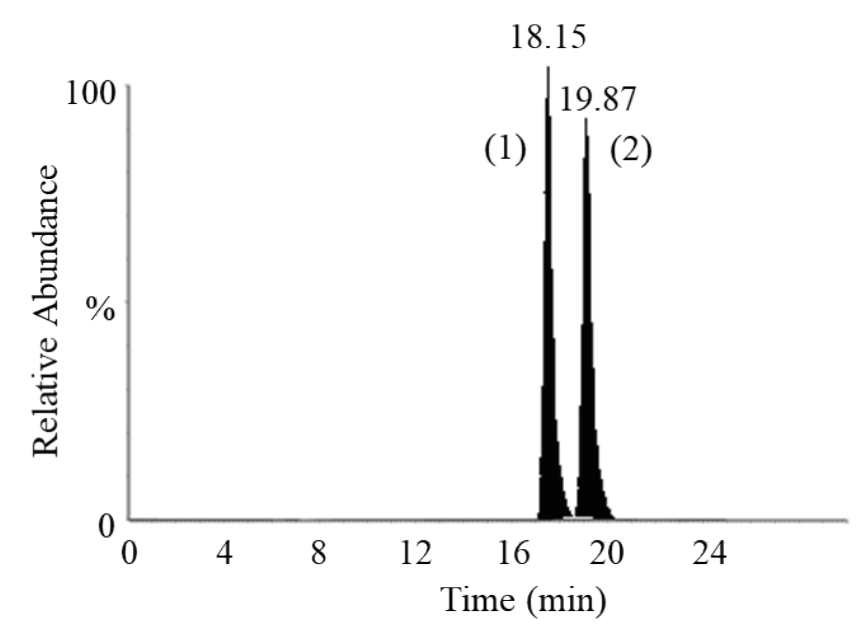

Fig. 4. Representative mass chromatogram of FMOC-synephrine in health foods No.7. Peak (1): FMOC- $d$-synephrine, Peak (2): FMOC-l-synephrine.

\section{Conclusions}

We developed an enantiomeric analytical method that uses LC/TOF-MS for the detection of synephrine. By performing FMOC derivatization of synephrine and using TCI Chiral MB-S as the chiral column, good mutual separation of FMOC-synephrine enantiomers was achieved.

The analytical method was applied to the analysis of synephrine-containing health foods to determine the enantiomeric excess. Approximately half of the products showed high enantiomeric excess of the $l$-isomer, whereas some products contained the racemate. For products containing the racemate, it was speculated that chemically synthesized synephrine was used, or that the $l$-isomer was converted into the $d$-isomer during the manufacturing process.

We conclude that this method can determine synephrine enantiomers in health foods, and is expected to be one of the useful analytical methods for evaluating product quality.

\section{References}

[1] Wheaton, T. A.; Stewart, I. Lloydia 1970, 33, 244-254.

[2] Carpéné, C.; Galitzky, J.; Fontana, E.; Atgié, C.; Lafontan, M.; Berlan, M. N.-S. Arch. Pharmacol. 1999, 359, 310-321.

[3] Fugh-Bergman, A.; Myers, A. Exp. Biol. Med. 2004, 229, 698-704.

[4] Calapai, G.; Firenzuoli, F.; Saitta, A.; Squadrito, F.; Arlotta, M. R.; Costantino, G.; Inferrera, G. Fitoterapia 1999, 70, 586-592.

[5] Marcus, D. M.; Grollman, A. P. Science 2003, 301, 1669-1671.

[6] Pellati, F.; Benvenuti, S.; Melegari, M. J. Pharm. Biomed. Anal. 2005, 37, 839-849.
[7] Pellati, F.; Benvenuti, S.; Melegari, M.; Firenzuoli, F. J. Pharm. Biomed. Anal. 2002, 29, 1113-1119.

[8] Brown, C. M.; McGrath, J. C.; Midgely, J. M.; Muir, A. G. B.; O’Brien, J. W.; Thonoor, C. M.; Williams, C. M.; Wilson, V. G. Br. Br. J. Pharmacol. 1988, 93, 417-429.

[9] Jordan, R.; Midgery, J. M.; Thonoor, C. M.; Williams, C. M. J. Pharm. Pharmacol. 1987, 39, 752-754.

[10] Pellati, F.; Benvenuti, S.; Yoshizaki, F.; Melegari, M. J. Sep. Sci. 2006, 29, 641-649.

[11] Kusu, F.; Matsumoto, K.; Takamura, K. Chem. Pharm. Bull. 1995, 43, 1158-1161.

[12] Kusu, F.; Matsumoto, K.; Arai, K.; Takamura, K. Anal. Biochem. 1996, 235, 191-194.

[13] Hashimoto, K.; Yasuda, T.; Ohsawa, K. J. Chromatogr. 1992, 623, 386-389.

[14] Roman, M. C.; Betz, J. M.; Hildreth, J. J. AOAC Int. 2007, 90, 68-81.

[15] Schaneberg, B. T.; Khan, I. A. Pharmazie 2004, 59, 819-823.

[16] Ganzera, M.; Lanser, C.; Stuppner, H. Talanta 2005, 66, 889-894.

[17] Hurlbut, J. A.; Carr, J. R.; Singleton, E. R.; Faul, K. C.; Madson, M. R.; Storey, J. M.; Thomas, T. L. J. AOAC Int. 1998, 81, 1121-1127.

[18] Niemann, R. A.; Gay, M. L. J. Agric. Food Chem. 2003, 51, 5630-5638.

[19] Juaristi, E. Introduction to stereochemistry and conformational analysis Wiley, New York, 1991, p.132.

[20] Hamase, K.; Morikawa, A.; Ohgusu, T.; Lindner, W.; Zaitsu, K. J. Chromatogr. A 2007, 1143, 105-111.

[21] Kohama, J.; Saito, K.; Sakamoto, H.; Iwasaki, Y.; Ito, R.; Horie, M.; Nakazawa, H. Bunseki Kagaku 2007, 56, 1019-1024.

[22] Saito, K.; Kohama, J.; Sakamoto, Y.; Iwasaki, Y.; Ito, R.; Horie, M.; Nakazawa, H. J. AOAC Int. 2011, 94, 482-486.

[23] Pellati, F.; Cannazza, G.; Benvenuti, S, J. Chromatogr. A 2010, 1217, 3503-3510.

[24] Tsuda, M., Macromol. Chem. 1964, 72, 174-182. 